\title{
Extended Dynamic Transmission Power Control Algorithm in Wireless Body Sensor Network Systems
}

\author{
Woosik Lee ${ }^{1}$, Min Choi ${ }^{2}$ and Namgi Kim ${ }^{1 *}$ \\ ${ }^{1}$ Dept. of Computer Science, Kyonggi Univ. Korea \\ ${ }^{2}$ School of Info. and Comm. Eng., Chungbuk National Univ., Korea \\ \{wslee,ngkim\}@kyonggi.ac.kr,mchoi@cbnu.ac.kr
}

\begin{abstract}
In the wireless body sensor network system (WB-SNS), saving energy in sensor nodes is a high priority. As an energy-saving method for sensor nodes, the transmission power control (TPC) algorithm has been developed. The TPC technique is a method of saving energy by adjusting the transmission power of the radio chip inside a sensor node. Numerous TPC algorithms exist, but in this paper, the dynamic TPC algorithm was analyzed intensively. The existing dynamic TPC algorithm utilizes a linear equation to find the optimal transmission power level (TPL). However, the existing dynamic TPC algorithm may operate well when the channel condition is good but it could estimate the TPL inaccurately due to the generation of an incorrect linear equation when the channel conditions are bad. Therefore, to solve the problem described above, this paper proposes an extended dynamic TPC algorithm that can estimate errors earlier based on slope values, which can be extracted at the time of generation of the linear equation, and estimate transmission power better by reutilizing the existing transmission power to create a more accurate equation. Through an experiment, this paper also proves that the extended dynamic TPC algorithm showed better performance compared to the existing dynamic TPC algorithm.
\end{abstract}

Keywords: body sensor network systems, dynamic transmission power control, closed loop system, wearable sensors, wireless sensor network

\section{Introduction}

The wireless body sensor network system (WB-SNS) is one of the promising technologies for the future and, to date, many relevant studies have been conducted [1-6]. In particular, body sensor networks represent a potentially great technology that can be applied in the area of U-Health care. In such a WB-SNS, a number of sensor nodes are arranged internally or externally on a person's body as well as on clothing to gather specified information. However, these sensor nodes are very small and operated by batteries so that energy use is significantly restricted. Furthermore, if a sensor in the WB-SNS is placed within the body, replacing a battery becomes much more difficult. In summary, a sensor in the body sensor network should operate in an energy-efficiency way. As a typical method for increasing the energy efficiency of sensor nodes, the technique of the TPC algorithm has been developed [7-10]. Many TPC algorithms are available [11-16] but in this paper, the dynamic TPC algorithm, which finds the optimal transmission power level (TPL) quickly using the linear equation, is discussed intensively [16]. The dynamic TPC algorithm produces a linear equation using two

* Corresponding author: Namgi Kim 
signal strength values measured in the received packet to find the optimal TPL. Using the produced equation, the dynamic TPC algorithm directly finds the optimal TPL. However, since the condition of a channel can be dynamically changed in a WB-SNS environment [17], it is difficult to produce the correct equation to find the optimal TPL. Due to this problem, when an incorrect equation is produced, a sensor node cannot find the optimal TPL and unnecessary energy waste or packet loss can result. To solve this problem, this paper proposes an extended dynamic TPC algorithm method. The proposed algorithm is an extended version of the dynamic TPC algorithm. It can discover incorrect slope generation error earlier during the equation generation and create a new equation. Therefore, the proposed method can reduce inaccurate transmission power estimation. To prove the superiority of the extended dynamic TPC algorithm, this paper demonstrated its better performance compared to the existing dynamic TPC algorithm through an experiment.

\section{Related Work}

Because a radio chip in a sensor node controls the TPL, the TPC algorithm is a technique to improve the energy efficiency of a sensor node by finding the optimal TPL. In the existing sensor network area, the TPC algorithm has been actively studied. However, in the case of the TPC algorithm used in the sensor network environment, effective systems can be developed because sensors are placed in fixed locations and the surrounding environment varies little. That is, the TPC algorithm that was not highly sensitive to the change in environment worked well in the general existing sensor network area. However, in a WB-SNS environment, human movement or a sensor's location can be changed dynamically and thus the TPC algorithm, which worked well in the sensor network environment, may not work well nor respond quickly to the environment change. Therefore, it is highly problematic if the TPC algorithm for a general sensor network environment is applied to the WB-SNS environment directly.

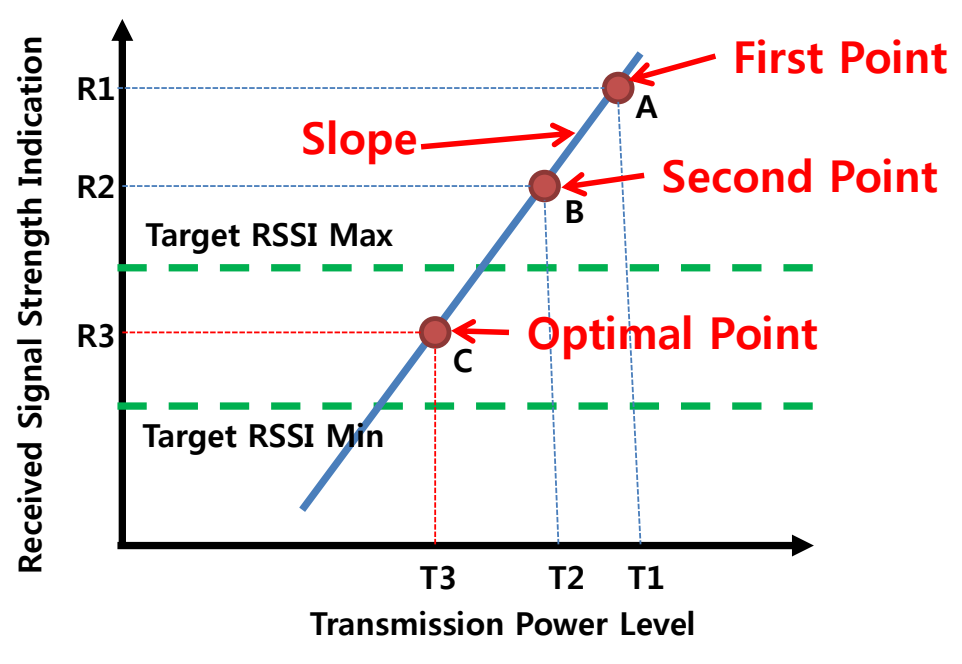

\section{Figure 1. Transmission power level assignment of the dynamic TCP algorithm}

As a typical TPC algorithm in the WB-SNS, linear [18], binary [19], and dynamic algorithms [16] have been developed. Among these various TPC algorithms, this paper intensively analyzed the dynamic TPC algorithm. The dynamic TPC algorithm searches 
the optimal TPL quickly using the linear equation. Figure 1 shows the detailed power assignment method of the dynamic TPC algorithm. In the figure, the $\mathrm{X}$ axis is a power level while the $\mathrm{Y}$ axis is the received signal strength indicator (RSSI) value representing the received signal strength. In the figure, the first point means a point where the RSSI value is R1 when a sensor node performs transmission of a packet to T1 power for the first time while the second point means a point where the RSSI value of the packet is R2 when the second packet is transmitted to T2 power. Using these two points, the first point and the second point, the dynamic TPC algorithm creates a linear equation having a specific slope. Finally, using the produced linear equation, a sensor node sets T3 of the TPL as the optimal transmission power value and transmits a packet. As a result, $\mathrm{C}$ point where the RSSI value is $\mathrm{R} 3$ can be determined.

\section{Extended Dynamic Transmission Power Control}
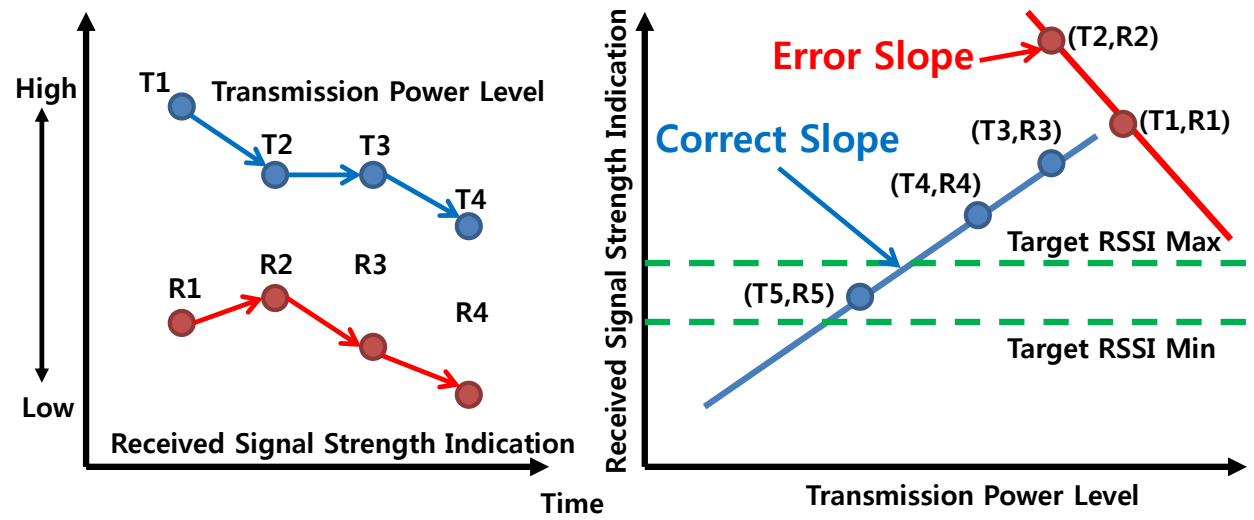

\section{Figure 2. Transmission power level assignment of the extended dynamic TPC algorithm}

Figure 2 shows the transmission power assignment method of the extended dynamic TPC algorithm proposed in this paper. In the figure, the left side graph shows the changes of the TPL and the RSSI while the right side graph shows the linear equation generation according to the TPL and the RSSI. In the left graph, X axis represents time, and T1, T2, T3, and T4 inside the graph represent the TPLs as to time while R1, R2, R3, and R4 show the RSSI values of the transmitted packets with the corresponding TPLs. In the figure, when a TPL continues from T1 to T2, the RSSI value increases from R1 to R2 according to the TPL. However, in the static channel environment, when a TPL decreases, the RSSI value shall decrease also, in general. In other words, when a TPL at T1 is reduced to T2, if the channel condition does not change, the R2 value shall be lower than the R1 value. The extended dynamic TPC algorithm can predict this incorrect state earlier through the slope of the linear equation. The reason for this is because the slope will be positive if the RSSI values change correctly as the transmission power changes. Therefore, when an incorrect RSSI value occurs in the extended dynamic TPC algorithm, the accurate equation can be found by retransmission using the previous TPLs for accurate linear equation generation. The right-side graph shows how the extended dynamic TPC algorithm finds the linear equation accurately. In the graph, the values of $(\mathrm{T} 1, \mathrm{R} 1)$ and $(\mathrm{T} 2, \mathrm{R} 2)$, which represent the TPL and the RSSI value were used in order for the extended dynamic TPC algorithm to produce a linear equation. However, in the case that the slope became negative, an incorrect linear equation 
was produced. Therefore, the extended dynamic algorithm retransmits a packet with T3 TPL, which was the same TPL as T2. Thereby, it obtains (T3, R3) and (T4, R4) using one level lower to produce an accurate linear equation. The extended dynamic TPC algorithm that produced more the accurate linear equation uses this equation at the next packet transmission time to obtain the RSSI value of R5 point and T5, which is a more accurate TPL.

\section{Comparison of Dynamic and Extended Dynamic Transmission Power Control}

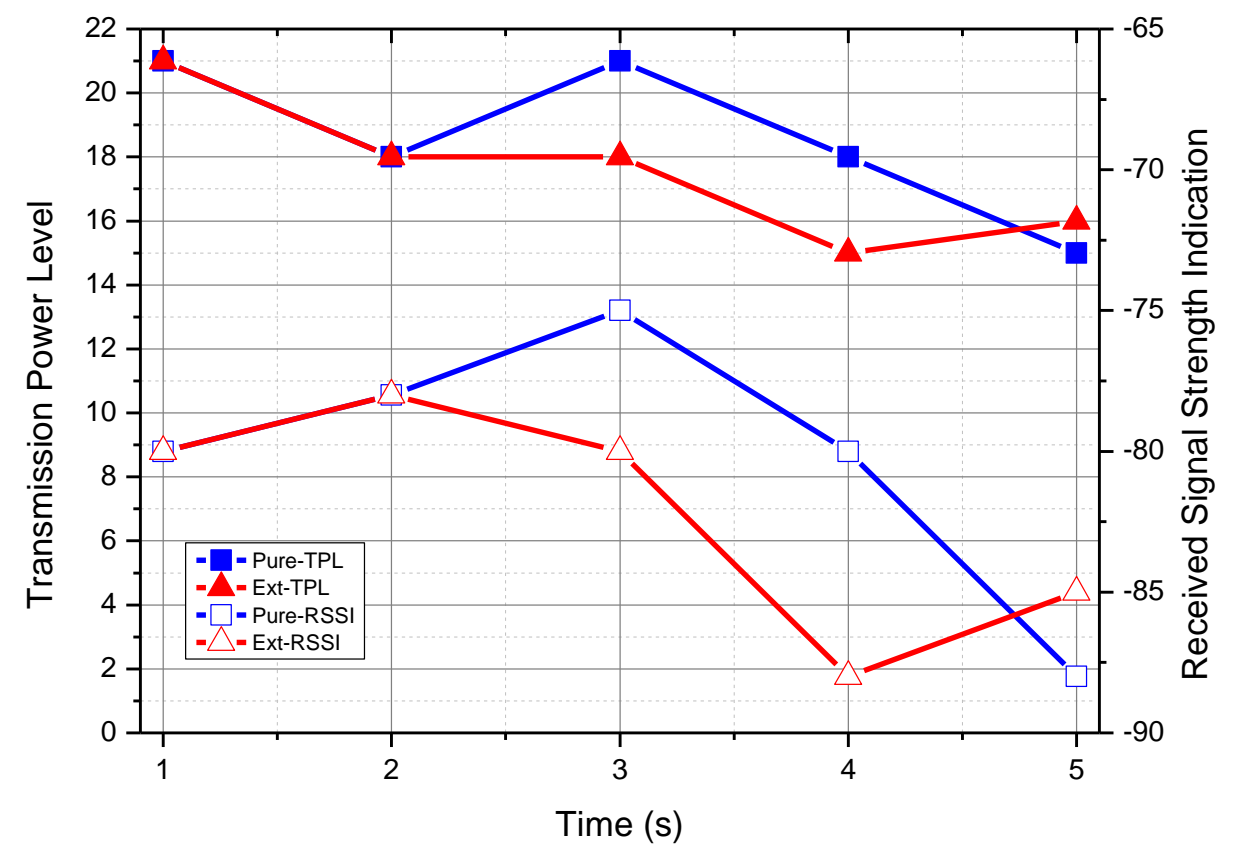

\section{Figure 3. Comparison of pure dynamic TPC algorithm and extended dynamic TPC algorithm}

Figure 3 shows the result of the experiment using the pure dynamic TPC algorithm and the extended dynamic TPC algorithm as time passed. In the figure, the $\mathrm{X}$ axis represents time and the Y axis represents the TPL and RSSI value. In the figure, as time passed, the pure dynamic TPC algorithm and the extended dynamic TPC algorithm operated the same up to the first two slots. That is, transmission was done with the maximum power level at first and the next transmission was done with one level lower transmission level. However, as shown in the figure, at 2 second, the RSSI value did not decrease but increased. At this time, the pure dynamic TPC algorithm produced an inaccurate equation so that transmission with the maximum TPL occurs at the next step, thereby wasting unnecessary energy. In contrast, the extended dynamic TPC algorithm did not produce an inaccurate equation but sent a retransmission with the previous power level to create a more accurate equation. In the graph, at the third slot time, the dynamic TPC algorithm performed transmission with the maximum TPL of 21, whereas the extended dynamic TPC algorithm performed transmission of a packet with a TPL of 18. Then, at the fifth and last slot time, both of the pure dynamic TPC algorithm and the extended dynamic TPC algorithm performed packet transmission with similar TPLs. The reason for this is that both algorithms produced an accurate linear equation at the third and fourth slot times. However, at the third and fourth slots, the pure dynamic 
TPC algorithm produced an equation of higher TPL compared to the extended algorithm. So, the pure dynamic TPC algorithm had conclusively more energy consumption. If this case happens rarely, it should not be a critical problem. But, due to bad channel condition, if this case occurs frequently, it will frequently create unnecessary energy consumption.

\section{System Architecture and Experimental Setup}

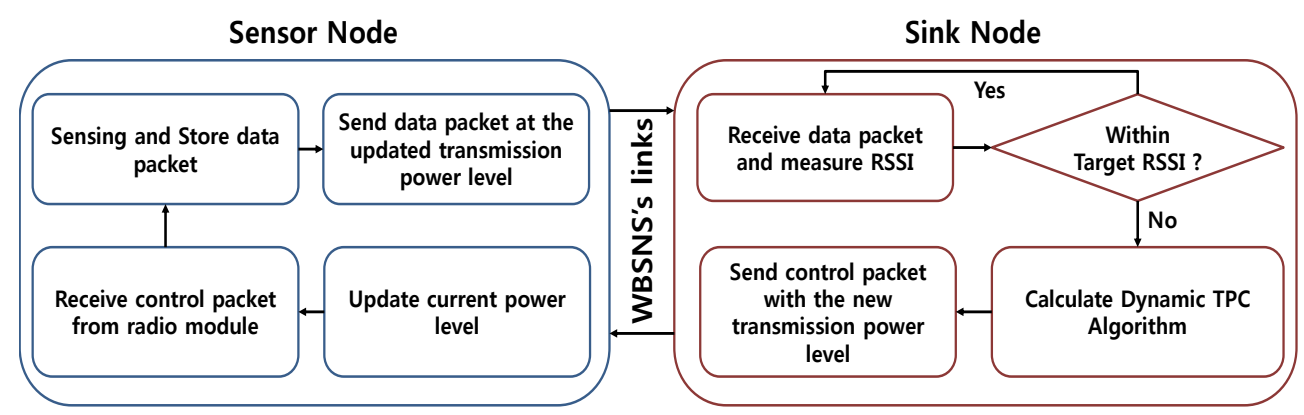

Figure 4. TPC closed-loop mechanism

Figure 4 shows the system architecture of the sensor node and sink node for the TPC closed-loop mechanism. The overall operation of the system architecture is as follows: In the figure, the left-side block represents a sensor node that performs transmission of a data packet while the right side means a sink node that receives the data packet. A sensor node plays the role of packet transmission with various TPLs while a sink node performs the finding of the next TPL based on the RSSI values of the packet received from the sensor node. For example, if a sensor node transmits a data packet with a specific power level, a sink node receives the data packet and measures the received signal strength. If the received signal strength measured is not located within the acceptable range, a sink node re-finds the TPL of the next data packet using the TPC algorithm. Then, the recalculated TPL value is inserted into the control packet, thereby transmitting this control packet to the sensor node. Here, the sensor node receives the control packet and changes the current TPL. This procedure is performed iteratively.

Figure 5 shows the location where this experiment was conducted as well as the sensor's location and inner structure. In the figure, the upper left end shows the hallway where the experiment of a lattice structure was conducted. While the upper right end shows the sensor that is attached to a human body. Further, the lower end shows for the structure how the sensor node stores the received signal strength value via packet transmission/receiving followed by delivering them to a computer. To analyze the overall operation more directly, a sink node collects a packet from a sensor node to store this in the EEPROM. Then, the stored packet is transmitted to a gateway node that is directly connected to a computer. Here, the gateway node performs only the delivery of the received packet to a host computer. Then, the host computer collects the packets and refines the packets as required by the experiment. Finally, the refined packets are utilized to run the simulator. 

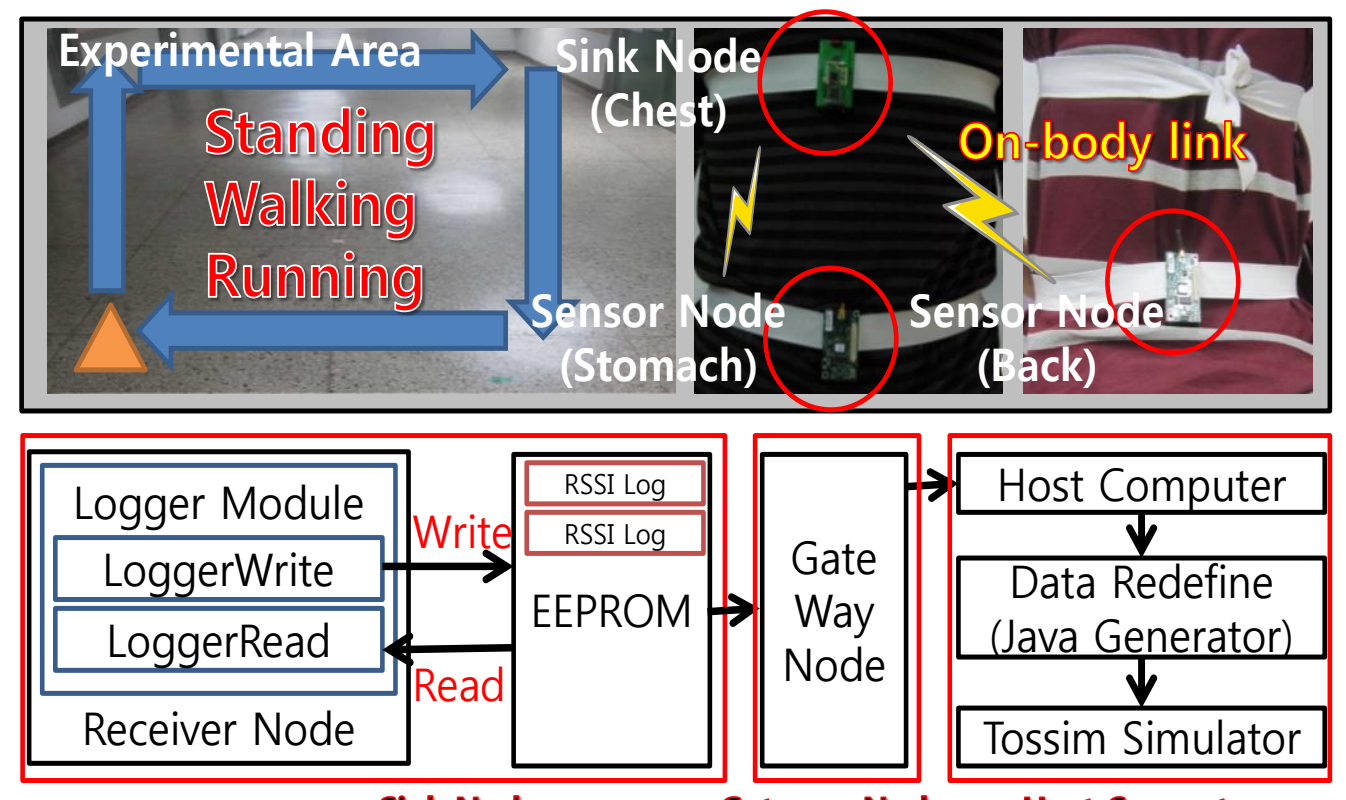

Sink Node

Gateway Node

Host Computer

\section{Figure 5. Experimental area and sensor deployment and system architecture}

\section{Experimental Result}

This paper performed the experiment repeatedly as the sensor locations and the number of received data packets was set up all the same to compare the performance of the TPC algorithms. In particular, all the experiments except for comparison of error generation were done using 1,000 data packets and the entire energy consumption of the sensor node was analyzed by considering retransmission.

Figure 6 shows the number of errors of linear equation as time passed in the pure dynamic TPC algorithm and the extended dynamic TPC algorithm when the channel condition was unstable. In the figure, the $\mathrm{X}$ axis represents time and the $\mathrm{Y}$ axis is the number of errors of the equation. As shown in the figure, the number of errors of the equation in the pure dynamic TPC algorithm and the extended dynamic TPC algorithm was the same as 0 but the difference increased as time elapsed. In particular, at 8,000 seconds, the extended dynamic TPC algorithm generated fewer equation errors by $14 \%$ (50 errors) compared to that of the pure dynamic TPC algorithm. Through this result, we verified that the extended dynamic TPC algorithm generated fewer errors as time passed compared to the pure dynamic TPC algorithm as well as increasing energy efficiency. 


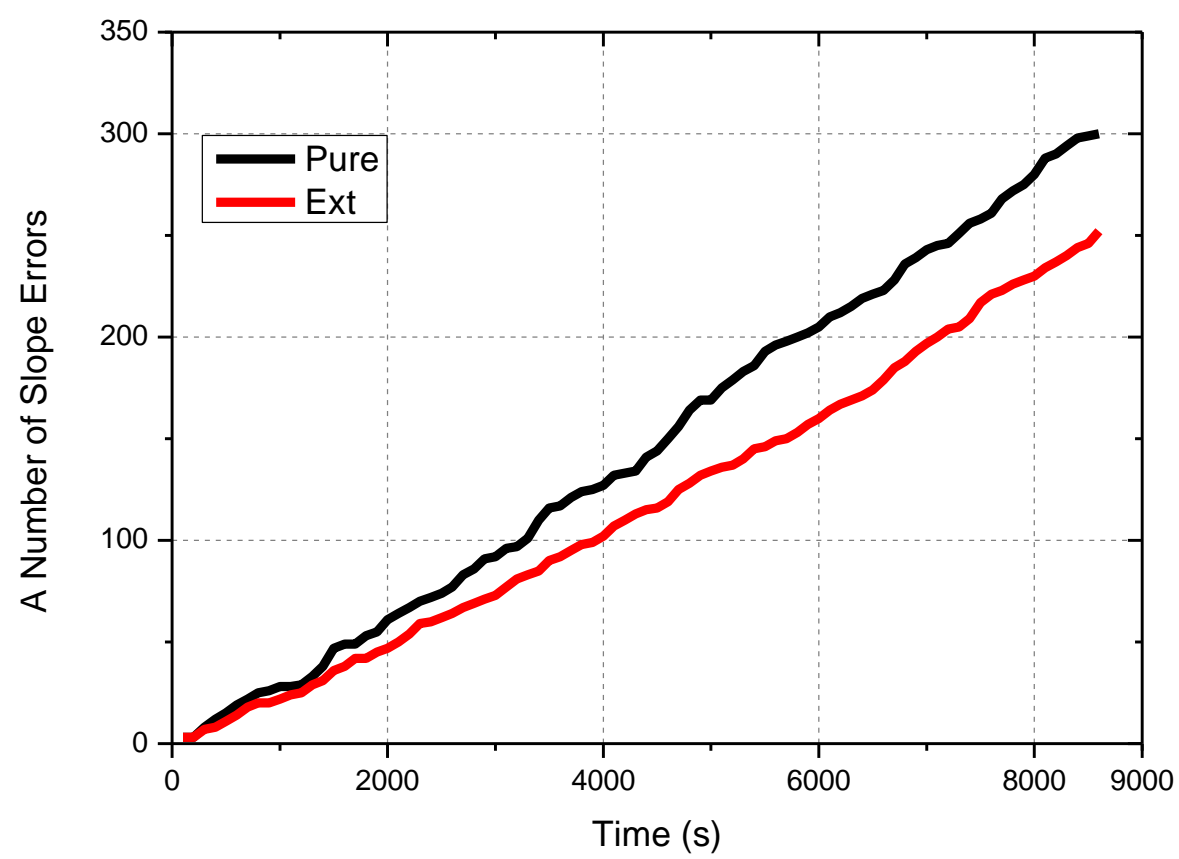

Figure 6. A number of slope errors

Figure 7 shows the packet transmission success rate of the pure dynamic TPC algorithm and the extended dynamic TPC algorithm. In the figure, SM means that a sensor was placed on the stomach while BK means that a sensor was placed on the back. Further, SD represented body movement in a standing state while WK and RN represented body movement in the walking and running states respectively. As can be seen in the figure, when a sensor was placed on the stomach, the pure dynamic TPC algorithm and the extended dynamic TPC algorithm showed their packet transmission success rates as nearly the same as $99 \%$, mostly. Further, when a sensor was placed on the back, the packet transmission success rate was $99 \%$ for both of the algorithms. The reason for this was because the channel condition was good in the standing state. However, when a sensor was placed on the back while the state of body movement was walking or running, the extended dynamic TPC algorithm showed a better packet transmission success rate than the pure dynamic TPC algorithm. Through this result, as the channel condition becomes worse, the packet transmission success rate of the pure dynamic TPC algorithm decreases further.

Figure 8 shows the entire energy consumption amount of the pure dynamic TPC algorithm and the extended dynamic TPC algorithm. In the figure, the $\mathrm{X}$ axis represents the sensor location and the person's movement while the $\mathrm{Y}$ axis represents the entire energy consumption amount. When a sensor was placed on the stomach, the dynamic TPC algorithm and the extended dynamic TPC algorithm showed the same energy consumption amount. The reason for this was because the channel condition was excellent thereby producing few errors. Since the case of a sensor placed on the back in the standing state had a good channel condition, both algorithms showed the same result. However, in the case of a sensor placed on the back while the person's movement was 
walking or running, the extended dynamic TPC algorithm had better energy efficiency than the pure dynamic TPC algorithm. The reason for this was because the pure dynamic TPC algorithm produced incorrect liner equations periodically, thereby estimating an inaccurate TPL. Through these results, we can conclude that the performance of the existing pure dynamic TPC algorithm could be worse as the channel condition becomes worse.

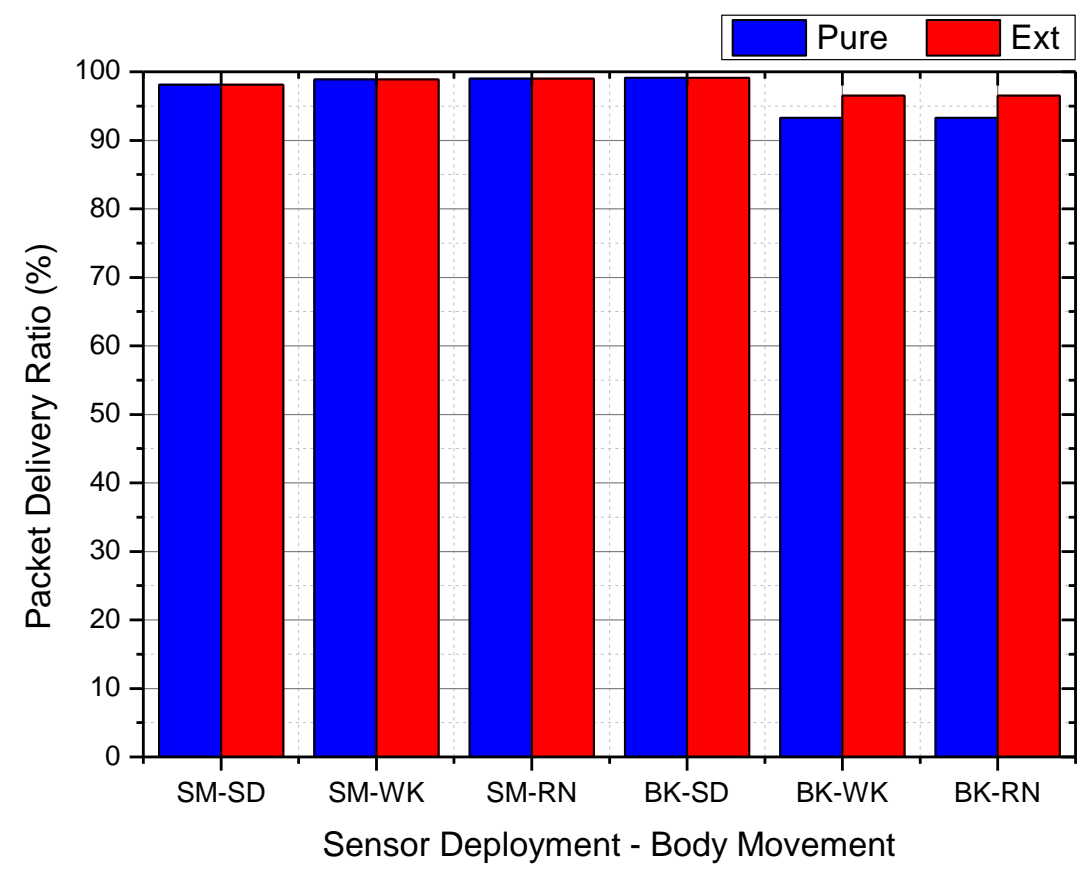

Figure 7. Packet delivery ratio

Earlier, we discussed the pure dynamic TPC algorithm and the extended dynamic TPC algorithm. The result showed that when a sensor was placed on the stomach in the standing, walking, and running states where the channel condition was excellent, the existing dynamic TPC algorithm and the extended dynamic TPC algorithm showed the same result. On the contrary, when a sensor was placed on the back in the walking and running state where the channel condition was bad, the extended dynamic TPC algorithm had around a 3\% better packet transmission success rate and around a $2 \%$ better energy efficiency than those of the existing dynamic TPC algorithm. Moreover, we found that the more time passed, the larger the equation error rate showed in the existing dynamic TPC algorithm. Based on these results, we have concluded that the performance of the extended dynamic TPC algorithm proposed in this paper is better than the existing dynamic TPC algorithm as the channel condition becomes worse. 


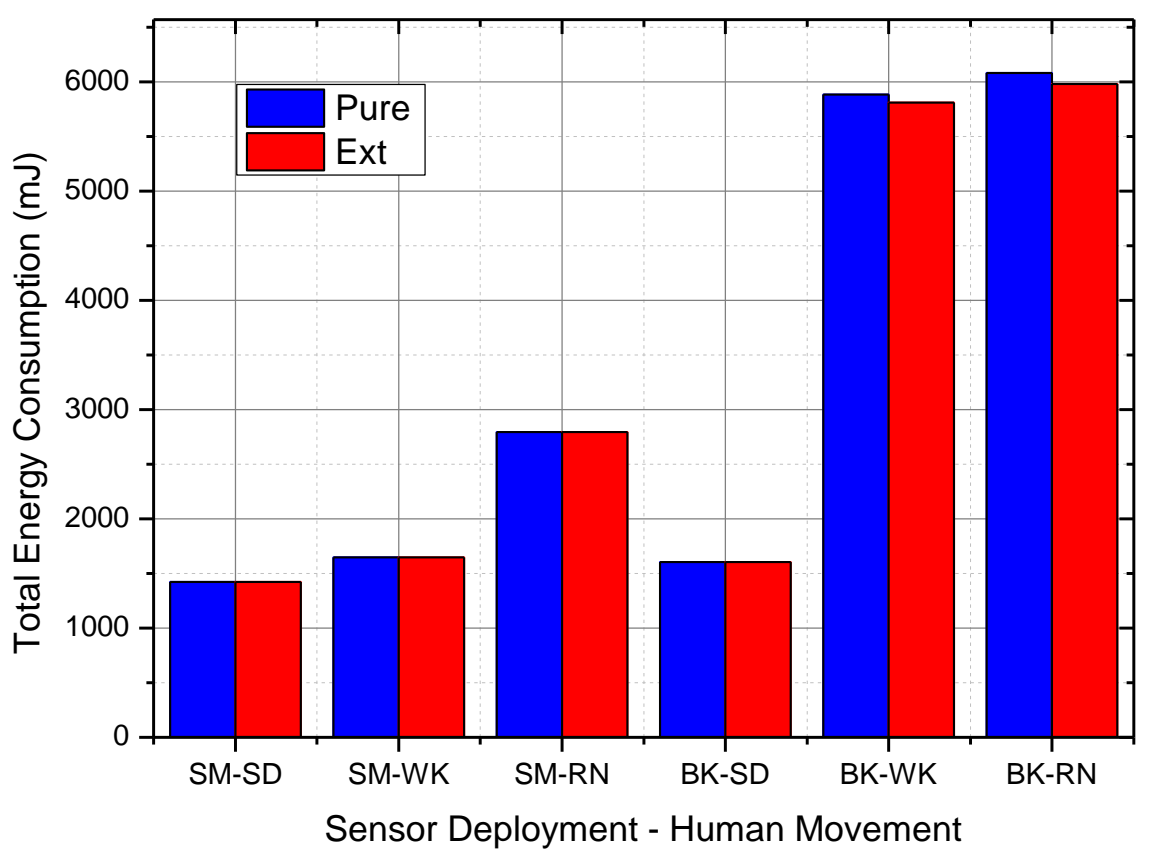

Figure 8. Total energy consumption

\section{Conclusion}

In the WB-SNS environment, channel condition changes dynamically according to the sensor's location and body movement. Therefore, both static and dynamic channel conditions need to be taken into consideration for a sensor node to increase energy efficiency through the TPC algorithm. In particular, this paper considered the dynamic TPC algorithm that can estimate a TPL quickly using the equation in the WB-SNS environment. The existing dynamic TPC algorithm estimates a TPL quickly by producing linear equations periodically. It works very well in a good channel condition. However, when the channel condition is bad, it produces incorrect equations, thereby frequently estimating an inaccurate TPL. To solve this problem, this paper reduced these equation generation errors by reusing the previous TPL if an error occurs during the time of equation generation. This paper also showed through an experiment that the extended dynamic TPC algorithm had better energy efficiency as well as a better packet transmission success rate compared to the existing dynamic TPC algorithm. We also found that the extended dynamic TPC algorithm had fewer equation errors compared to the dynamic TPC algorithm, thereby producing more energy gains.

In the future, we will propose a novel TPC algorithm that can estimate channel condition quickly without equation generation while increasing energy efficiency. We will also consider a measure for increasing energy efficiency through a fusion system by utilizing various sensors used in the body sensor area.

\section{Acknowledgements}

This research was supported by Basic Science Research Program through the National Research Foundation of Korea (NRF) funded by the Ministry of Science, ICT \& Future Planning (grant number 2012R1A1A1002133)

\section{References}


[1] V. Shnayder, B. Chen, K. Lorincz, T. R. F. Fulford-Jones and M. Welsh, "Sensor Network for Medical Care", TR-08-05, Harvard Univ., (2005).

[2] T. Gao, T. Massey, L. Selavo, D. Crawford, B. Chen, K. Lorincz, V. Shnayder, L. Hauenstein, F. Dabiri, J. Jeng, A. Chanmugam, D. White, M. Sarrafzadeh and M. Welsh, "The advanced Health and disaster aid network: A lightweight wireless medical system for triage”, IEEE Trans. Biomedical circuits and Sys., vol. 1, (2007) September, pp. 203-216.

[3] A. Milenkovic, C. Otto and E. Jovanov, "Wireless sensor networks for personal health monitoring: Issues and an implementation", Comp. Comm., vol. 29, (2006) August, pp. 2521-2533.

[4] S. W. Franklin and S. E. Rajan, "Personal area network for biomedical monitoring systems using human body as a transmission medium", International Journal of Bio-Science and Bio-Technology, vol. 2, no. 2, (2010) June, pp. 2-3-28.

[5] J. Jung, K. Ha and J. Lee, "Wireless body area network in a ubiquitous healthcare system for physiological signal monitoring and health consulting", International Journal of Signal Processing, Image Processing and Pattern Recognition, vol. 1, no. 1, (2008) December, pp. 47-54.

[6] K. Miller and S. Sankaranarayanan, "Policy based agents in wireless body sensor mesh networks for patient health monitoring", International Journal of U- and E-Service, Science and Technology, vol. 2, no. 4, (2009) December, pp. 37-50.

[7] C. Li, H. B. Li and R. Kohno, "Reservation-based dynamic TDMA protocol for medical body area networks", IEICE Trans. Communication, vol. 92, (2009) February, pp. 387-395.

[8] S. Ullah, R. Islam, A. Nessa, Y. Zhong and K. S. Kwak, "Performance analysis of preamble based TDMA protocol for wireless body area network", Journal of Communication Software System, vol. 4, (2008) September, pp. 222-226.

[9] G. Fang and E. Dutkiewicz, "BodyMAC: Energy efficient TDMA-based MAC protocol for wireless body area networks", IEEE ISCIT, (2009) September, pp. 1455-1459.

[10] J. Kim, I. Song, E. Jang and S. Choi, "A dynamic duty cycle MAC algorithm for wireless body area networks", International Journal of Bio-Science and Bio-Technology, vol. 4, no. 2, (2012) June, pp. 83-92.

[11] C. N. Pelin and C. Erdal, "PCSMAC, A power controlled sensor-MAC protocol for wireless sensor networks", Wireless Sens. Net., (2005).

[12] W. Ye, J. Heidemann and D. Estrin, "An energy-efficient MAC protocol for wireless sensor networks", Infocom, vol. 3, (2002) June, pp. 1567-1576.

[13] L. H. A. Correia and J. M. S. Nogueira, "Transmission power control in MAC protocols for wireless sensor networks", ACM/IEEE MSWiM, (2005) October, pp. 282-289.

[14] S. Lin, J. Zhang, G. Zhou, L. Gu, T. He and J. Stankovic, "ATPC: Adaptive transmission power control for wireless sensor networks”, ACM SenSys, (2006) November, pp. 223-236.

[15] W. Lee and N. Kim, "A study of transmission power control algorithms in wireless body sensor systems", Proceedings of Advanced Science and Technology Letters, (2013) April.

[16] M. Quwaider, J. Rao and S. Biswas, "Body-posture-based dynamic link power control in wearable sensor networks", IEEE Communications Magazine, vol. 48, (2010) July, pp. 134-142.

[17] W. Lee, M. Choi and N. Kim, "Different Characteristics of Radio Modules in Wireless Body Area Networks", Lecture Notes in Computer Science, vol. 7513, (2012) September, pp. 308-314.

[18] D. Knuth, "The Art of Computer Programming", Sorting and Searching (3rd edition), Addison-Wesley (1997).

[19] T. H. Cormen, C. E. Leiserson, R. L. Rivest and C. Stein, "Introduction to algorithms (1st edition)", MIT Press and McGraw-Hill, (1990).

\section{Authors}

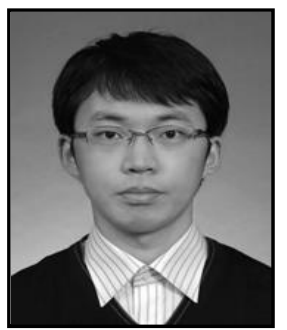

\section{Woosik Lee}

He received the B.S. degree in Computer Science from the Kyonggi University, Korea, in 2009, and the M.S. degree in the Computer Science from Kyonggi University in 2011. He is currently Ph.D. candidate in Computer Science from Kyonggi University. His research interests include wireless systems, sensor networks, and energy management protocols. 


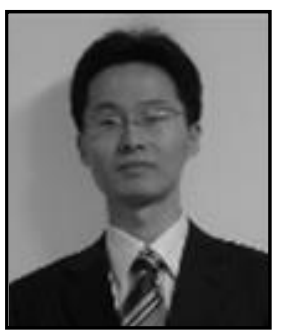

\section{Min Choi}

He received the B.S. degree in Computer Science from Kwangwoon University, Korea, in 2001, and M.S. and Ph.D. degrees in Computer Science from Korea Advanced Institute of Science and Technology (KAIST) in 2003 and 2009, respectively. From 2008 to 2010, he worked for Samsung Electronics as a Senior Engineer. Since 2011 he has been a faculty member of Department of Information and Communication of Chungbuk National University. His current research interests include embedded system, computer architecture, and mobile cloud.

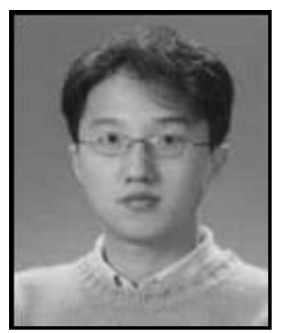

\section{Namgi Kim}

He received the B.S. degree in Computer Science from Sogang University, Korea, in 1997, and the M.S. degree and the Ph.D. degree in Computer Science from KAIST in 2000 and 2005, respectively. From 2005 to 2007, he was a research member of the Samsung Electronics. Since 2007, he has been a faculty of the Kyonggi University. His research interests include sensor system, wireless system, and mobile communication. 
International Journal of Control and Automation Vol.6, No.6 (2013) 